\title{
NUMERICAL METHODS AND ERROR ESTIMATES FOR A SINGULAR BOUNDARY-VALUE PROBLEM
}

\author{
P.M. LIMA ${ }^{1}$ and A.M. OLIVEIRA ${ }^{2}$ \\ ${ }^{1}$ Instituto Superior Técnico, Departamento de Matemática \\ Av. Rovisco Pais, 1049-001 Lisboa, Portugal \\ E-mail: plima@math.ist.utl.pt \\ ${ }^{2}$ Faculdade de Ciências e Tecnologia, Departamento de Matemática \\ Quinta da Torre, 2829-516 Caparica, Portugal \\ E-mail: amfoliveira@yahoo.com
}

Received September 9, 2002

\begin{abstract}
In this paper we analyze a class of equations of the form $y^{\prime \prime}(x)=-g(x) x^{p}(y(x))^{q}$ where $p$ and $q$ are real parameters satisfying $p>-1, q<-1$ and $g$ is a positive and continuous function on $[0,1]$. We search for positive solutions which satisfy the boundary conditions $y^{\prime}(0)=y(1)=0$.

Numerical approximations of the solution are obtained by means of a finite difference scheme and the asymptotic expansion of the discretization error is deduced. Some numerical examples are analyzed.
\end{abstract}

Key words: singular problems, finite differences scheme, asymptotic expansion.

\section{INTRODUCTION}

In the present paper we consider boundary-value problems for a generalized Emden-Fowler equation of the form

$$
y^{\prime \prime}(x)=-g(x) x^{p} y(x)^{q}, \quad 0<x<1
$$

where $p$ and $q$ are known real constants and $g$ is continuous and positive on $[0,1]$. We shall look for positive solutions of this equation on the interval $[0,1]$, which satisfy certain boundary conditions. 
A problem of this type was analyzed in [2], with $p=1, q<0$ and $g(x)=$ $-1 / q$. This problem arises in non-newtonian fluid mechanics. The solution of the considered problem has a singularity at $x=1$ which results from the fact that $q$ is negative and the solution must satisfy

$$
\lim _{x \rightarrow 1^{-}} y(x)=0
$$

The asymptotic behavior of the solutions to this problem near the singularity was analyzed in detail and it was shown that there exists an oneparametric family of solutions of the considered equation which satisfy the required boundary condition at the singularity. The asymptotic expansion of this family was then used to approximate the solutions near the singularities. In this way, we can replace the singular boundary condition at $x=1$ by a non-singular one at a certain point $x=1-\delta$. Then we can approximate numerically the solutions of the considered family, using standard numerical methods for initial-value problems and, by the shooting method, we can find the specific solution which satisfies the boundary condition at $x=0$, which in this case has the form

$$
\lim _{x \rightarrow 0^{+}} y^{\prime}(x)=0 \text {. }
$$

A different approach for the same problem was developed in [5] and [6]. In these papers, the nonlinear problem is first reduced to a sequence of linear ones, by means of monotone iterative procedures, using a method proposed by Mooney (see [9] and [10]). Then the linear problems are approximated by means of finite difference schemes. In the cited papers, the convergence of the iterative methods was analyzed and the asymptotic behavior of the solutions near the singularity was described.

In [8] the considered approach was generalized for a wider class of problems. It was shown that the considered methods can be applied not only to the case $p=1$, but also for other values of $p$, such that $p>-1$.

Recently, in [7] we have analysed a BVP for equation (1.1) with boundary conditions

$$
\lim _{x \rightarrow 1^{-}} y(x)=\lim _{x \rightarrow 0^{+}} y(x)=0 .
$$

In particular, we have shown for this problem how the convergence order of the finite difference scheme depends on $p$ and $q$. The problem that we shall consider here has many common aspects with that one, in particular the asymptotic behavior of the solution, as $x \rightarrow 1$, is the same. Therefore, in the present paper, we shall often refer to the results of [7] an will not repeat the proofs.

In [4] and [3] a similar method was used to approximate the solutions of singular Emden-Fowler equations, with $p<0$ and $q>0$ (in this case the solution has a singularity at $x=0$ ). In those papers the asymptotic behavior of the 
solutions near the singularity was studied and asymptotic error expansions were obtained.

The main purpose of the present paper is to provide a detailed error analysis for the considered methods, in the case where $q<0$, when the solution is singular at $x=1$ or at both end points. We derive asymptotic error expansions which are valid for a large range of values of $p$ and $q$ in the case of boundary conditions (1.2), (1.3). We show that these error expansions agree with the previous numerical experiments and use them as a basis for applying extrapolation methods such as the $E$-algorithm.

In Section 2, we review the results obtained in [5] and [8] on upper and lower solutions for the considered problem. In Section 3, we define the iterative methods and analyze the asymptotic behavior of the iterates near the singularities. In Section 4, we obtain the asymptotic error expansions, using a method similar to the one which was used in [3]. In Section 5 some numerical examples are presented and extrapolation algorithms, based on the asymptotic error expansions, are used to accelerate the convergence. Finally, in Section 6 we present the main conclusions of this work.

\section{LOWER AND UPPER SOLUTIONS}

As defined in previous papers (see for instance [5] and [8]), a lower solution (respect. upper solution) of BVP (1.1)-(1.2)-(1.3) is a function $\widetilde{y}(x) \in$ $C^{2}(] 0,1[) \cap C([0,1])$ that satisfies the set of conditions

$$
\left\{\begin{array}{cc}
\widetilde{y}^{\prime \prime}(x)+g(x) x^{p}(\widetilde{y}(x))^{q} \geq 0 & (\text { respect. } \leq 0) \\
\widetilde{y}^{\prime}(0) \geq 0 \quad(\text { respect. } \leq 0) & ; \quad \widetilde{y}(1)=0
\end{array}\right.
$$

We are interested in upper and lower solutions which satisfy (2.1), and whose asymptotic behavior near the singularity is close to the one of the exact solution.

In order to fit the above conditions, we shall look for upper and lower solutions of the form

$$
\widetilde{y}(x)=C\left(1-x^{p+2}\right)^{\gamma},
$$

where $C$ and $\gamma$ are adjustable parameters.

By setting

$$
\gamma=\frac{2}{1-q}
$$

we assure that $\widetilde{y}(x)$ is asymptotically equivalent to $k y(x)$ when $x \rightarrow 1$, where $k$ is a positive constant.

Defining $\gamma$ as above, by some basic computations, it is possible to find positive constants $C_{m}$ and $C_{M}$, such that, if $C \leq C_{m}$ (respect. $\geq C_{M}$ ), then $\widetilde{y}(x)$ is a lower solution (respect. upper solution). 
Table 1.

Bounds for the coefficient $C$ of $(2.2)$. Here $\omega_{1}=(1-\gamma)(p+2), \omega_{2}=p+1$, $g_{m}=\min _{x \in[0,1]} g(x), g_{M}=\max _{x \in[0,1]} g(x)$.

\begin{tabular}{cc}
$y_{l}$ & $y_{u}$ \\
$C_{m}=\left(\frac{g_{m}}{\gamma(p+2) \omega_{1}}\right)^{\frac{\gamma}{2}}$ & $C_{M}=\left(\frac{g_{M}}{\gamma(p+2) \omega_{2}}\right)^{\frac{\gamma}{2}} \quad$ if $0<\gamma<\frac{1}{p+2}$ \\
$C_{m}=\left(\frac{g_{m}}{\gamma(p+2) \omega_{2}}\right)^{\frac{\gamma}{2}}$ & $C_{M}=\left(\frac{g_{M}}{\gamma(p+2) \omega_{1}}\right)^{\frac{1}{2}} \quad$ if $\frac{1}{p+2} \leq \gamma<1$ \\
\hline
\end{tabular}

With the purpose of simplifying the notation, we shall denote by $y_{l}(x)$ (respect. by $\left.y_{u}(x)\right)$ a lower solution (respect. upper solution).

The values of $C_{m}$ and $C_{M}$ may be synthesized in the Table 1

A remarkable particular case arises when

$$
g_{m}=g_{M}=\text { const }=a \quad \gamma=\frac{1}{p+2}
$$

that is, when $2 p+q=-3$. In this case, we note that $C_{m}=C_{M}$, meaning that the lower and upper solution of the problem coincide and give the exact solution:

$$
y(x)=\left(\frac{a}{p+1}\right)^{\frac{\gamma}{2}}\left(1-x^{p+2}\right)^{\gamma} .
$$

In particular, if we set $p=1$ and $q=-5$ we get the solution mentioned in [2].

Many theoretical results about nonlinear boundary-value problems for second order equations are applicable to the considered problem. For example, according to Theorem 7.5 of [12] we can assure that this problem has an unique solution, for all the considered values of the parameters.

\section{ITERATIVE METHODS AND ASYMPTOTIC BEHAVIOR OF THE SOLUTIONS}

\subsection{Monotone iterative schemes}

As it was pointed out in [5] and [6], the nonlinear problem (1.1) - (1.3) may be transformed into a sequence of linear ones, using two monotone iterative schemes, based either on the Picard or the Newton methods. Since both mentioned methods converge to the exact solution, for $k$ sufficiently large, the asymptotic behavior of $y_{k}$ near the singularity will be similar to the asymptotic behavior of $y$; in this paper, for the asymptotic analysis we shall consider the 
Picard iterative scheme, which in the case (1.1) - (1.3) has the form

$$
\begin{aligned}
L y_{k+1}(x) & =y_{k+1}^{\prime \prime}(x)+q g(x) x^{p}\left(y_{l}(x)\right)^{q-1} y_{k+1}(x) \\
& =g(x) x^{p} y_{k}(x)\left(q\left(y_{l}(x)\right)^{q-1}-\left(y_{k}(x)\right)^{q-1}\right), \\
y_{k+1}^{\prime}(0) & =y_{k+1}(1)=0, \quad 0<x<1, \quad k \geq 0,
\end{aligned}
$$

and $y_{l}(x)$ is a lower solution of the form (2.2), with $C=C_{m}$, where $C_{m}$ is defined as in Table 1.

As it was is shown in [5], if we take as $y_{0}$ a lower solution, the iterative scheme (3.1) converges upwards to the exact solution.

\subsection{Asymptotic behavior of the Picard iterates}

For the error analysis that we shall present in the next Section we need some results about the asymptotic behavior of the Picard iterates. These results are obtained in the same way as it was done in [7], and therefore we shall not go here into details. First we shall analyze the homogeneous equation, associated to (3.1):

$$
y^{\prime \prime}(x)+b(x) y(x)=0,0<x<1,
$$

where

$$
b(x)=q\left(C_{m}\right)^{q-1} g(x) x^{p}\left(1-x^{p+2}\right)^{-2} .
$$

For all the considered values of $p$ and $q$, equation (3.2) has a regular singularity at $x=1$. For $p<0$, the equation has an irregular singularity at $x=0$, but if $p=-m / n$ this irregular singularity may be transformed into a regular one by means of the variable transformation $x=t^{1 / n}$. In both cases, the roots of the indicial equation associated to (3.2) can be computed. Let $\rho_{1}$ and $\rho_{2}$ be the roots of the indicial equation as $x \rightarrow 1$, then we have $\rho_{1}+\rho_{2}=1$, $\rho_{1}<0, \rho_{2}>1$. Using these roots we can express two independent solutions of (3.2) in the form of series and analyse their behavior. This analysis leads us to the following lemma.

Lemma 3.1. Let us consider equation (3.2), where $q<-1, p\rangle-1$ and $g(x)$ are such that the roots of the indicial equation do not differ by an integer not an integer; if $p<0$, let $p$ be rational.

Then, there exists an unique solution $\alpha$ of (3.2), such that

$$
\lim _{x \rightarrow 0^{+}} \alpha(x)=1 ; \lim _{x \rightarrow 0^{+}} \alpha^{\prime}(x)=0 ;
$$

and an unique solution $\beta$ which satisfies

$$
\lim _{x \rightarrow 1^{-}}(1-x)^{-\rho_{2}} \beta(x)=1
$$


These solutions are independent and such that

$$
\begin{gathered}
\lim _{x \rightarrow 1^{-}}(1-x)^{-\rho_{1}} \alpha(x)=\alpha_{0} ; \\
\lim _{x \rightarrow 0^{+}} \beta(x)=\beta_{0},
\end{gathered}
$$

where $\alpha_{0}$ and $\beta_{0}$ are positive finite numbers.

Now the classical method of the Green function enables us to express the Picard iterate $y_{k+1}$, which is the solution of (3.1), in terms of the preceding iterate $y_{k}$ and the functions $\alpha, \beta$, described in Lemma 3.1. According to this method we can write

$$
y_{k+1}(x)=\int_{0}^{1} G(x ; s) f_{k}(s) d s=\frac{\beta(x)}{\alpha(1)} \int_{0}^{x} \alpha(s) f_{k}(s) d s+\frac{\alpha(x)}{\alpha(1)} \int_{x}^{1} \beta(s) f_{k}(s) d s
$$

where

$$
f_{k}(x)=g(x) x^{p} y_{k}(x)\left[q\left(C_{m}\right)^{q-1}\left(1-x^{p+2}\right)^{-2}-\left(y_{k}(x)\right)^{q-1}\right]
$$

Knowing the asymptotic behavior of $y_{0}(x)$, which is a lower solution, formula (3.6) and Lemma 3.1 enable us to obtain the asymptotic properties of the iterates $y_{k}, k \geq 1$. These properties are described by the following theorem.

Theorem 3.1. Let us consider the sequence of problems (3.1) with boundary conditions $y_{k+1}^{\prime}(0)=y_{k+1}(1)=0$, and $p>-1$; if $p<0$, let $p$ be rational.

If $y_{0}(x)$ is a lower solution of the form (2.2), then there exist constants $y_{k+1,0}$ and $\overline{y_{k+1,0}}$ such that

$$
y_{k+1}(x)=y_{k+1,0}+O\left(x^{p+2}\right), \quad k=0,1, \ldots, \text { as } x \rightarrow 0^{+} \text {, }
$$

and

$$
y_{k+1}(x)=\overline{y_{k+1,0}}(1-x)^{\gamma}+t_{k+1}(x), \quad k=0,1, \ldots, \text { as } x \rightarrow 1^{-} \text {, }
$$

where $\gamma=\frac{2}{1-q}$ and $t_{k+1}(x)=o\left((1-x)^{\gamma}\right)$.

Moreover, if $\gamma+1<\rho_{2}$, where $\rho_{2}$ is the greatest root of the indicial equation at $x=1$, we have $t_{k+1}(x)=O\left((1-x)^{\gamma+1}\right)$. 


\section{DISCRETIZATION METHODS AND ASYMPTOTIC ERROR EXPANSIONS}

Let $\wp=\left\{x_{i}=i h\right\}_{i=0, \ldots, N}$ be an uniform grid, with $h=1 / N$, and

$$
y_{k+1}(x ; h): \wp \rightarrow \mathbb{R}_{0}^{+}
$$

the discrete function which results from evaluating $y_{k+1}$ at $\wp$.

In order to discretize equations (3.1), we shall substitute the derivative $y_{k+1}^{\prime \prime}(x)$ by the corresponding second-order central difference

$$
\delta^{2} y_{k+1}\left(x_{i} ; h\right)=\frac{1}{h^{2}}\left(y_{k+1}\left(x_{i+1} ; h\right)-2 y_{k+1}\left(x_{i} ; h\right)+y_{k+1}\left(x_{i-1}, h\right)\right) .
$$

Therefore, for each $k$, we shall solve a linear system of difference equations of the form

$$
L^{h} \widetilde{y}_{k+1}=\delta^{2} \widetilde{y}_{k+1}\left(x_{i} ; h\right)+b\left(x_{i}\right) \widetilde{y}_{k+1}\left(x_{i} ; h\right)=\widetilde{f}_{k}\left(x_{i} ; h\right),
$$

where

$$
\widetilde{f}_{k}\left(x_{i} ; h\right)=\phi\left(x_{i}\right) \widetilde{y}_{k}\left(x_{i} ; h\right)\left(q\left(C_{m}\right)^{q-1}\left(1-x_{i}^{p+2}\right)^{-2}-\left(\widetilde{y}_{k}\left(x_{i} ; h\right)\right)^{q-1}\right),
$$

in order to find the unknown vector $\left(\widetilde{y}_{k+1}\left(x_{i} ; h\right)\right)_{i=0, \ldots, N}$ which approximates each continuous function $y_{k+1}(x), k \geq 0$.

The full system must include two equations that are the discrete analogs of the boundary conditions

$$
\left\{\begin{array}{l}
\widetilde{y}_{k+1}(0 ; h)=\widetilde{y}_{k+1}(h ; h) \\
\widetilde{y}_{k+1}(1 ; h)=0, \quad k \geq 0 .
\end{array} .\right.
$$

Let us define, for each iterate $\widetilde{y}_{k+1}(x ; h)$, the discretization error

$$
\theta_{k+1}(x ; h)=\widetilde{y}_{k+1}(x ; h)-y_{k+1}(x),
$$

for $x \in[0,1], h>0$.

We shall first consider $k=0$. As in [7], we shall derive the asymptotic expansion of $\theta_{1}(x ; h)$ as $h \rightarrow 0$. If $\varepsilon_{1}(x ; h)$ is the consistency error of the finite-differences scheme, for the first iterate $y_{1}(x ; h)$, defined as

$$
\varepsilon_{1}(x ; h)=\delta^{2} y_{1}(x ; h)-y_{1}^{\prime \prime}(x),
$$

then we have

$$
L^{h} \theta_{1}(x ; h)=-\varepsilon_{1}(x ; h) .
$$


In order to obtain an asymptotic expansion of $\theta_{1}(x ; h)$ we shall use the same method as in [7]. The outline of this method is the following. Since $\theta_{1}(x ; h)$ is a solution of the difference equation (4.7) and $L^{h}$ is an approximation of the linear differential operator $L$, we look for $\theta_{1}(x ; h)$ in the form

$$
\theta_{1}(x ; h)=\mu_{1}(h) \alpha(x)+\mu_{2}(h) \beta(x)+\theta_{1 p}(x ; h)
$$

where $\alpha$ and $\beta$ are the functions referred in Lemma 3.1 (solutions of the homogeneous equation), $\theta_{1 p}$ is a particular solution of (4.7), $\mu_{1}$ and $\mu_{2}$ are certain functions of $h$.

From the asymptotic properties of $y_{1}$, described by Theorem 3.1, certain conditions follow that $\theta_{1}(x ; h)$ must satisfy. In particular, making $x=h$ in (4.8) and considering the boundary condition $y_{1}^{\prime}(0)=0$, we obtain

$$
\mu_{1}(h) \alpha(h)+\mu_{2}(h) \beta(h)+\theta_{1 p}^{\prime}(h ; h)=\overline{B_{0}} h^{p+1}+o\left(h^{p+1}\right) ;
$$

making $x=1-h$ and considering the boundary condition at $x=1$, we have

$$
\mu_{1}(h) \alpha(1-h)+\mu_{2}(h) \beta(1-h)+\theta_{1 p}(1-h ; h)=\overline{C_{0}} h^{\gamma}+O\left(h^{\gamma+1}\right) .
$$

On the other hand, a particular solution $\theta_{1 p}(x ; h)$ of $(4.7)$ may be computed in the form

$$
\theta_{1 p}(x ; h)=\sum_{i=1}^{m-1} \omega_{2}(x) h^{2 i}+O\left(h^{2 m}\right),
$$

where the $\omega_{2} i$ coefficients are the solutions of certain boundary value problems. By analyzing these coefficients we may conclude that $\theta_{1 p}$ satisfies the conditions

$$
\begin{gathered}
\theta_{1 p}^{\prime}(h ; h)=\bar{\theta}_{0} h^{p+1}+o\left(h^{p+1}\right), \quad \text { as } \quad h \rightarrow 0 \\
\theta_{1, p}(1-h ; h)=\theta_{1,0} h^{\gamma}+O\left(h^{\gamma+1}\right) .
\end{gathered}
$$

From the results of the previous Section, it follows that, for some constant $\alpha_{1}$, we have $\alpha(1-h)=\alpha_{1} h^{\rho_{1}}(1+o(1))$, as $h \rightarrow 0$, where $\rho_{1}$ is the least root of the indicial equation, associated to (3.2). Hence,from (4.10) we may conclude that

$$
\mu_{1}(h)=\mu_{1,0} h^{\gamma-\rho_{1}}+o\left(h^{\gamma-\rho_{1}}\right) .
$$

In the same way, using (4.9) and (4.11) we may conclude that

$$
\mu_{2}(h)=\mu_{2,0} h^{p+1}+o\left(h^{p+1}\right) .
$$

Finally, if we substitute (4.13) and (4.14) into (4.8), we obtain the desired expression of $\theta_{1}(x ; h)$ :

$$
\theta_{1}(x ; h)=\widehat{\theta_{1,0}} h^{m_{1}}+o\left(h^{m_{1}}\right),
$$


where

$$
m_{1}=\min \left(\gamma-\rho_{1}, p+1\right) .
$$

In order to derive analogous asymptotic error expansions for iterates $k=$ $2,3, \ldots$ we may follow a similar procedure as in [7].

Omitting further details, we would conclude that, when $m_{1} \leq 1$, the order of the main term of the discretization error remains the same as in the first iterate. Howener, when $m_{1}>1$, the expected convergence order of the subsequent iterates is 1 .

In the next Section, this conclusion is confirmed by the numerical examples.

\section{NUMERICAL RESULTS}

With the help of a MATHEMATICA code, a wide range of numerical tests were performed for a large range of values of $p$ and $q$ (for the same value of $p$ several values of $q$ were studied). Our purpose is to compare the theoretical convergence order estimates with the experimental ones.

We focused our attention on the cases $g(x)=1$ and $g(x)=e^{x}$. The iterative process (4.2) was carried out with four different step sizes $h_{1}=\frac{1}{100}$; $h_{i+1}=\frac{h_{1}}{2^{i}}, i=1,2,3$. For each $h_{i}$ the iterative process (4.2) was stopped at the iterate $k_{i}^{*}$ that satisfies

$$
\left(\sum_{1}^{N}\left|y_{k_{i}^{*}+1}\left(x_{j} ; h_{i}\right)-y_{k_{i}^{*}}\left(x_{j} ; h_{i}\right)\right|^{2}\right)^{\frac{1}{2}} \leq 10^{-7} .
$$

Based on these numerical results, two different estimates of the convergence order of scheme (4.2) - (4.4), one of the estimates being a local one, $e_{k}\left(x_{j}\right)$, computed at a fixed point $x_{j}(j \in\{1, \ldots, N-1\})$, and the other, $r_{k}$ being a global estimate, in terms of error norms. For each iterate these estimates are defined as

$$
e_{k}\left(x_{j}\right)=\log _{2} \frac{\left|y_{k}\left(x_{j} ; h_{3}\right)-y_{k}\left(x_{j} ; h_{2}\right)\right|}{\left|y_{k}\left(x_{j} ; h_{4}\right)-y_{k}\left(x_{j} ; h_{3}\right)\right|}
$$

and

$$
r_{k}=\log _{2} \frac{\max _{j=1, \ldots, N-1}\left|y_{k}\left(x_{j} ; h_{3}\right)-y_{k}\left(x_{j} ; h_{2}\right)\right|}{\max _{j=1, \ldots, N-1}\left|y_{k}\left(x_{j} ; h_{4}\right)-y_{k}\left(x_{j} ; h_{3}\right)\right|} .
$$

Tables 2 (where $g(x)=1$ ) and Tables 3 (where $g(x)=e^{x}$ ) confirm that, for all fixed values of $p \geq 0$ and for several values of $q$, there exist no significant discrepancies between the theoretical orders of convergence deduced in the previous Section and its estimates given by formulae (5.2) and (5.3).

The main term of the asymptotic error expansion remains the same even if the condition $\gamma+1<\rho_{2}$ is not satisfied. 
Table 2.

Experimental order of convergence, $e_{1}($ at $x=0.5)$ and $r_{1}$, and the theoretical convergence order, for some values of $p$.

\begin{tabular}{|c|c|c|c|c|c|c|c|}
\hline $\mathbf{p}=-0.7$ & $e_{1}(0.50)$ & $r_{1}$ & $m_{1}$ & $\mathbf{p}=-\mathbf{0 . 3}$ & $e_{1}(0.50)$ & $r_{1}$ & $m_{1}$ \\
\hline$q=-1.1$ & 0.3658 & 0.3617 & 0.3000 & $q=-1.1$ & 0.7291 & 0.7168 & 0.7000 \\
\hline$q=-1.5$ & 0.3824 & 0.3700 & 0.3000 & $q=-1.5$ & 0.7620 & 0.7261 & 0.7000 \\
\hline$q=-2.0$ & 0.4202 & 0.3912 & 0.3000 & $q=-2.0$ & 0.8009 & 0.7385 & 0.7000 \\
\hline$q=-2.5$ & 0.4565 & 0.4067 & 0.3000 & $q=-2.5$ & 0.8264 & 0.9007 & 0.7000 \\
\hline$q=-3.0$ & 0.4906 & 0.4177 & 0.3000 & $q=-3.0$ & 0.8514 & 0.9014 & 0.7000 \\
\hline $\mathbf{p}=0.0$ & $e_{1}(0.50)$ & $r_{1}$ & $m_{1}$ & $\mathbf{p}=0.5$ & $e_{1}(0.50)$ & $r_{1}$ & $m_{1}$ \\
\hline$q=-1.1$ & 1.0440 & 1.0149 & 1.0000 & $q=-1.1$ & 1.5159 & 1.3056 & 1.3897 \\
\hline$q=-1.5$ & 1.0779 & 1.0275 & 1.0000 & $q=-1.5$ & 1.3315 & 1.1479 & 1.2849 \\
\hline$q=-2.5$ & 1.0410 & 0.9473 & 1.0000 & $q=-2.5$ & 1.1379 & 1.0076 & 1.1236 \\
\hline$q=-3.5$ & 1.0024 & 0.9025 & 1.0000 & $q=-3.5$ & 1.0402 & 0.9309 & 1.0323 \\
\hline$q=-4.0$ & 1.0024 & 0.9034 & 1.0000 & $q=-4.5$ & 1.0059 & 0.9045 & 1.0000 \\
\hline $\mathbf{p}=1.0$ & $e_{1}(0.50)$ & $r_{1}$ & $m_{1}$ & $\mathbf{p}=\mathbf{2 . 0}$ & $e_{1}(0.50)$ & $r_{1}$ & $m_{1}$ \\
\hline$q=-1.5$ & 1.3570 & 1.1813 & 1.3247 & $q=-1.5$ & 1.4104 & 1.2220 & 1.3724 \\
\hline$q=-2.0$ & 1.2452 & 1.1004 & 1.2339 & $q=-2.0$ & 1.2986 & 1.1433 & 1.2847 \\
\hline$q=-2.5$ & 1.1741 & 1.0454 & 1.1680 & $q=-4.0$ & 1.1075 & 0.9947 & 1.1042 \\
\hline$q=-4.0$ & 1.0503 & 0.9453 & 1.0475 & $q=-6.0$ & 1.0274 & 0.9291 & 1.0250 \\
\hline$q=-6.0$ & 1.0023 & 0.9068 & 1.0000 & $q=-8.0$ & 1.0023 & 0.9092 & 1.0000 \\
\hline
\end{tabular}

However, for $p<0$ and when $q$ decreases, the experimental estimates of the convergence order become significantly higher than its expected value.

An explanation for this fact is that, for a fixed negative $p$, the solution $y(x)$ of (1.1) satisfies $\lim _{x \rightarrow 0^{+}} y(x)>1$, so $\lim _{q \rightarrow-\infty} y(0)^{q}=0$.

Therefore, since $y^{\prime \prime}(x)=g(x) x^{p} y(x)^{q}$, the effect of the singularity at $x=0$, due to $x^{p}$, is in some sense smoothened, and the result is an increasing of the experimental order of convergence.

Table 3.

Experimental order of convergence, $e_{1}($ at $x=0.5)$ and $r_{1}$, and the theoretical convergence order, for some values of $p$.

\begin{tabular}{|c|c|c|c|c|c|c|c|}
\hline $\mathbf{p}=-0.7$ & $e_{1}(0.50)$ & $r_{1}$ & $m_{1}$ & $\mathrm{p}=1.0$ & $e_{1}(0.50)$ & $r_{1}$ & $m_{1}$ \\
\hline$q=-1.1$ & 0.3609 & 0.3581 & 0.3000 & $q=-1.5$ & 1.8159 & 1.5606 & 1.8571 \\
\hline$q=-1.5$ & 0.3757 & 0.3649 & 0.3000 & $q=-2.0$ & 1.7593 & 1.5301 & 1.7995 \\
\hline$q=-2.0$ & 0.4015 & 0.3824 & 0.3000 & $q=-2.5$ & 1.7218 & 1.5075 & 1.7563 \\
\hline$q=-2.5$ & 0.4211 & 0.3948 & 0.3000 & $q=-4.0$ & 1.6526 & 1.4637 & 1.6747 \\
\hline$q=-3.0$ & 0.4365 & 0.4032 & 0.3000 & $q=-6.0$ & 1.6582 & 1.4803 & 1.6774 \\
\hline
\end{tabular}

Fig. 1 illustrates this fact for $g(x)=1$. The graphic on Fig.2 shows how the convergence order depends on $q$ for a fixed positive value of $p(p=0.75)$. In all examples concerning the case $g(x)=1$, for a fixed value of $p$ we have chosen some values of $q$ such that $q \neq-2 p-3$ ( we have not considered here 
the cases where the exact solution is known). The results obtained in the case $g(x)=e^{x}$ show that, when $p>0$ the form of $g$ does not affect the convergence order (as it could be expected from the theoretical values). However, when $p<0$, we obtain for $g(x)=e^{x}$ a higher convergence order than for $g(x)=1$, if the same values of $p$ and $q$ are considered (see for instance the Tables2 and $3 p=-0.7)$.

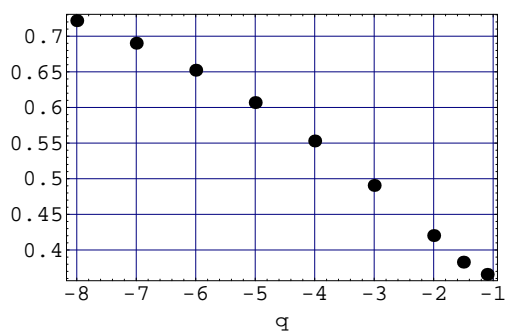

Figure 1. Plot of the estimate of the convergence order, $e_{1}(0.50)$, computed for several values of $q$. Here $p=-0.7$ and $g(x)=1$.

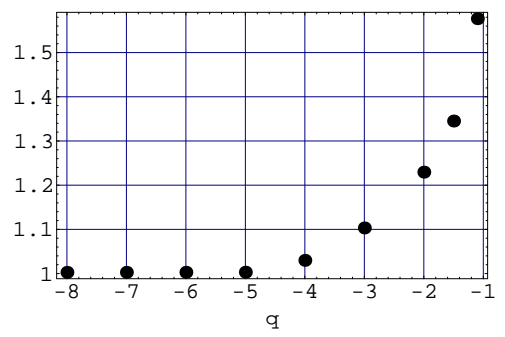

Figure 2. Plots of the estimate of the convergence order, $e_{1}(0.50)$, computed for several values of $q$. Here $p=-0.75$ and $g(x)=1$.

For each sequence of aproximations

$$
y_{k}\left(x_{j} ; h_{1}\right), y_{k}\left(x_{j} ; h_{2}\right), y_{k}\left(x_{j} ; h_{3}\right), y_{k}\left(x_{j} ; h_{4}\right),
$$

the E-algorithm may be applied to accelerate its convergence. The E-algorithm is a general extrapolation procedure introduced by C.Brezinski which is described, for example, in [1]. It generalizes classical methods like the Richardson extrapolation but it has the advantage that it can be applied to singular cases like the present ones, provided that an asymptotic error expansion is known.

Let the initial sequence be $S_{n}=E_{0}^{(n)}=y_{k_{n}^{*}+1}\left(x_{j} ; h_{n}\right), n=0,1,2, \ldots, n_{\max }$ and let us suppose that the discretization error allows an asymptotic expansion of the form

$$
y_{k_{i+1}^{*}}\left(x_{j} ; h_{n}\right)-y\left(x_{j}\right)=a_{1} g_{1}(n)+a_{2} g_{2}(n)+\cdots+a_{k} g_{k}(n) .
$$

Then, at each step of the E-algorithm, we obtain a new sequence $E_{k}^{(n)}$ of approximations. The number of steps that may be performed for a given sequence is the number of known terms of the asymptotic error expansion (5.5).

In $([3 ; 4 ; 7])$ we have observed that the E-algorithm provides a significant improvement of the accuracy, when asymptotic error expansions are known.

As an illustration of the efficiency of this process, when applied to our case, we selected two examples, where the exact solution is given by (2.4), that is, when $2 p+q=-3$ and $g(x)=1$. The results are displayed in Tables 4 and 5 . 
Table 4.

Components of the E-array in the case $p=-0.55, q=-1.9, g(x)=1$. In this case, the exact solution, up to 9 digits, is $y(0.5)=0.961792981$ and therefore the module of the absolute error of $E_{1}^{(0)}$ is approximately $6.13 \times 10^{-4}$.

\begin{tabular}{cccc}
\hline$i$ & $h_{i}$ & $E_{0}^{(i)}=y_{v^{*}+1}\left(0.50 ; h_{i}\right)$ & $E_{1}^{(i)}$ \\
\hline 0 & $\frac{1}{400}$ & 0.950761418 & 0.962406112 \\
1 & $\frac{1}{800}$ & 0.953881697 & \\
\hline
\end{tabular}

Table 5.

Components of the E-array in the case $p=1.2, q=-5.4, g(x)=1$. In this case, the exact solution, up to 9 digits, is $y(0.5)=0.852826787$ and the absolute error of $E_{1}^{(0)}$ is approximately $5.8 \times 10^{-7}$.

\begin{tabular}{cccc}
\hline$i$ & $h_{i}$ & $E_{0}^{(i)}=y_{v^{*}+1}\left(0.50 ; h_{i}\right)$ & $E_{1}^{(i)}$ \\
\hline 0 & $\frac{1}{400}$ & 0.852126852 & 0.852827370 \\
1 & $\frac{1}{800}$ & 0.852477111 & \\
\hline
\end{tabular}

In both cases, only the main term of the asymptotic error expansion is known; therefore, we use just two different stepsizes and perform only one step of the extrapolation process.

The effect of the convergence acceleration may then be seen by comparing the absolute error of the terms of $E_{1}^{(i)}$ with the absolute errors of $E_{0}^{(i)}$.

In both selected cases, $E_{0}^{(i)}=S_{i}=y_{k_{i}^{*}+1}\left(0.50 ; 2^{-i-2} 10^{-2}\right), i=0,1$.

In the first case, we have $p=-0.55$ and $q=-1.9$; therefore, from formula (4.15), we obtain $g_{1}(n)=\left(h_{n}\right)^{0.45}$.

In the second case, $p=1.2$ and $q=-5.4$; hence, from the same formula, we have $g_{1}(n)=h_{n}$.

As it was expected, the extrapolation procedure significantly improves the results. The higher order of convergence of the second example results in a higher accuracy in the value of $E_{1}^{(0)}$.

\section{CONCLUSIONS}

In this paper we have dealt with a class of singular boundary value problems for second order nonlinear ordinary differential equations. We have analyzed the dependence of the solution on two real parameters $p$ and $q$, and also on a certain regular function $g$. The theoretical analysis of the problem was based on its reduction to a monotone sequence of linear problems by means of the Picard method. Then we have obtained series expansions of the Picard iterates near the singularities at the endpoints. This approach enabled us 
to obtain information about the asymptotic behavior of the solution, which agrees with the results obtained in [2], for a particular case. For the numerical approximation of the linear problems we have applied the finite difference method. Then using the same technique as in [7], we have analyzed the discretization error of this method and obtained formulas for the convergence order. As it could be expected, this order depends on $p$ and $q$ and is closely related to the asymptotic behavior of the solution near the singularities. The lowest convergence order was obtained in the cases where $p$ is negative (in this case, we have shown that the convergence order is $p+1)$. When $p>0$, the convergence order is not less than 1 and depends strongly on $q$. These results were obtained theoretically ant then confirmed by numerical experiments.

Based on these asymptotic error expansions, convergence acceleration was carried out using the E-algorithm. The presented examples show that this method indeed improves the accuracy of the numerical results.

As it was pointed out in [6], the convergence of the finite-difference methods for singular boundary value problems may be significantly improved by introducing an adequate variable substitution. In some cases, this method provides more accurate results than the ones obtained in the present paper. However, the considered method of variable substitution is not applicable to all the considered cases, for example, it does not work when $p<0$. In such cases, the best way to obtain an accurate approximation is to apply the finite difference method to the original equation and improve the convergence by means of extrapolation, as proposed in the present paper.

\section{REFERENCES}

[1] C. Brezinski and M.R. Zaglia. Extrapolation Methods, Theory and Practice. NorthHolland, Amsterdam, 1991.

[2] A.L. Dyshko, M.P. Carpentier, N.B. Konyukhova and P.M. Lima. Singular problems for Emden-Fowler type second-order nonlinear differential equations. Comput. Maths. Math. Phys., 41, 557 - 580, 2001.

[3] P.M. Lima. Numerical methods and asymptotic error expansions for the Emden-Fowler equations. J. of Comp. and Applied Mathematics, 70, 245 - 266, 1996.

[4] P.M. Lima and M.P. Carpentier. Asymptotic expansions and numerical approximation of nonlinear degenerate boundary-value problems. Appl. Num. Math., 30, 93 - 111, 1999.

[5] P.M. Lima and M.P. Carpentier. Iterative methods for a singular boundary-value problem. J. of Comp. and Applied Mathematics, 111, 173 - 186, 1999.

[6] P.M. Lima and M.P. Carpentier. Numerical solution of a singular boundary-value problem in non-Newtonian fluid mechanics. Comp. Phys. Comm., 126, $114-120,2000$.

[7] P.M. Lima and A.M. Oliveira. Numerical methods for a singular boundary-value problem for a generalized Emden-Fowler equation. Applied Numerical Mathematics. (to appear)

[8] P.M. Lima and A.M. Oliveira. Aproximação de problemas de valores de fronteira singulares usando subsoluções e supersoluções. In: Tendências da Matemática Aplicadae Computacional (actas da XXII Conferência Nacional de Matemática Aplicada e Computacional), volume $1(2), 401-414,2000$. (in portuguese) 
[9] J.W. Mooney. A unified approach to the solution of certain classes of nonlinear boundary value problems using monotone iterations. Nonlinear Analysis, 3, 449 - 465, 1979.

[10] J.W. Mooney. Numerical schemes for degenerate boundary-value problems. J. Phys. $A, \mathbf{2 6}, \mathrm{L} 413$ - L421, 1993.

[11] J.W. Mooney. Solution of a Thomas-Fermi problem using linear approximants. Comput. Phys. Comm., 76, $51-57,1993$.

[12] A. Tineo. A comparison theorem for second order odes and applications to singular problems. Journal of Differential Equations, 16, 16 - 30, 1995.

\section{Singuliariuju kraštinių uždavinių skaitiniai metodai ir paklaidų iverčiai}

P.M. Lima, A.M. Oliveira

Nagrinëjama viena klasè antrosios eilès netiesiniu diferencialinių lygčių su kraštine sąlyga. Uždavinys yra singuliarusis viename arba abiejuose intervalo galuose. Siūlomas skaitinis metodas taikytinas atskiriems uždavinių klasès atvejams. Darbas tęsia ankstesnius autorių darbų tyrimus. Pateikti skaitinio eksperimento rezultatai, patvirtinantys teorinius įverčius. 